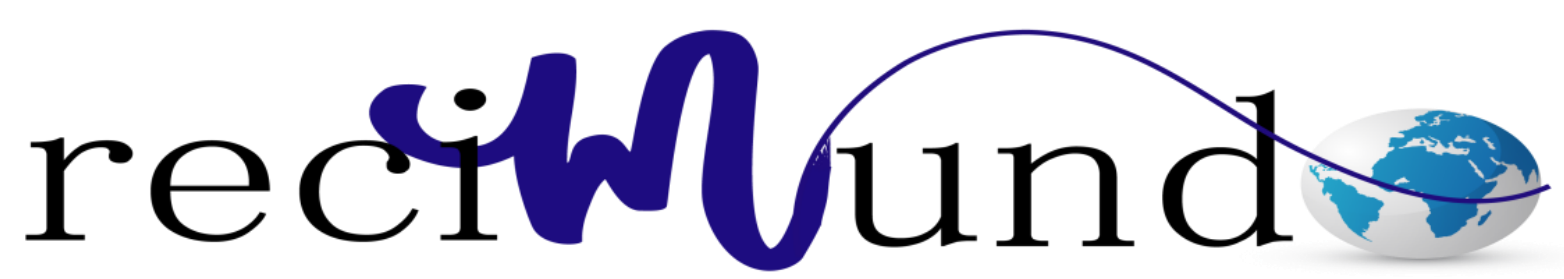

Revista Científica Mundo de la Investigación y el Conocimiento

Andrea Geraldine Castro Maquilón ${ }^{\text {a; }}$ Charles David Altamirano Olvera ${ }^{\text {b; }}$ Thalía Jocelyn Medina Checa ${ }^{\text {c; }}$ Juan Sebastián de Mora Flores ${ }^{\mathrm{d}}$

Bypass gástrico en el tratamiento de diabetes tipo 2 en la juventud

Gastric bypass in the treatment of type 2 diabetes in youth

Revista Científica Mundo de la Investigación y el Conocimiento. Vol. 3 núm.3, septiembre, ISSN: 2588-073X, 2019, pp. 145-162

DOI: 10.26820/recimundo/3.(3).septiembre.2019.145-162

URL: http://recimundo.com/index.php/es/article/view/515

Código UNESCO: 3205 Medicina Interna

Tipo de Investigación: Artículo de Revisión

Editorial Saberes del Conocimiento

Recibido: $15 / 05 / 2019$

Aceptado: 23/06/2019

Publicado: 30/09/2019

Correspondencia: andreacastroczs5@gmail.com

a. Médica; Investigador Independiente; Guayaquil, Ecuador; andreacastroczs5@ gmail.com

b. Médico; Investigador Independiente; Guayaquil, Ecuador; chardei2729@ hotmail.com

c. Médico; Investigador Independiente; Guayaquil, Ecuador; thalia j17e@ hotmail.com

d. Médico; Investigador Independiente; Guayaquil, Ecuador; sebastian_demora@hotmail.com 


\section{Bypass gástrico en el tratamiento de diabetes tipo 2 en la juventud}

Vol. 3, núm. 3., (2019)

Andrea Geraldine Castro Maquilón; Charles David Altamirano Olvera; Thalía Jocelyn Medina Checa; Juan Sebastián de Mora Flores

\section{RESUMEN}

La diabetes tipo 2, una vez que se hace referencia como la diabetes "del adulto", ahora se ha convertido en una amenaza formidable para la salud de los adolescentes obesos. Aunque existe una creciente evidencia sobre la epidemiología de la diabetes tipo 2 en jóvenes y sus consecuencias multisistémicas para la salud, las opciones de tratamiento se han quedado y la progresión de la enfermedad se produce incluso con tratamiento médico agresivo. El creciente interés en la aplicación de la cirugía bariátrica para los adolescentes con diabetes tipo 2 se ha convertido en parte debido a la evidencia que demuestra la mejoría o remisión en muchos adultos con diabetes después de la cirugía. En este artículo examinamos la carga de la diabetes tipo 2 en jóvenes, incluyendo sus complicaciones asociadas tipo, discuten los resultados y las complicaciones de la cirugía bariátrica en adolescentes con diabetes.

Palabras Claves: Cirugía bariátrica; Diabetes tipo 2; Tratamiento. 


\title{
Bypass gástrico en el tratamiento de diabetes tipo 2 en la juventud
}

Vol. 3, núm. 3., (2019)

Andrea Geraldine Castro Maquilón; Charles David Altamirano Olvera; Thalía Jocelyn Medina Checa; Juan Sebastián de Mora Flores

\begin{abstract}
Type 2 diabetes, once referred to as "adult" diabetes, has now become a formidable threat to the health of obese adolescents. Although there is growing evidence on the epidemiology of type 2 diabetes in young people and its multisystemic health consequences, treatment options have remained and the progression of the disease occurs even with aggressive medical treatment. The growing interest in the application of bariatric surgery for adolescents with type 2 diabetes has become part due to evidence that demonstrates improvement or remission in many adults with diabetes after surgery. In this article we examine the burden of type 2 diabetes in young people, including its associated type complications, discuss the results and complications of bariatric surgery in adolescents with diabetes.
\end{abstract}

Key Words: Bariatric surgery; Type 2 diabetes; Treatment. 


\section{Bypass gástrico en el tratamiento de diabetes tipo 2 en la juventud}

Vol. 3, núm. 3., (2019)

Andrea Geraldine Castro Maquilón; Charles David Altamirano Olvera; Thalía Jocelyn Medina Checa; Juan Sebastián de Mora Flores

\section{Introducción.}

La diabetes mellitus tipo 2 se considera una de las enfermedades crónicas con mayor impacto en la calidad de vida de la población mundial y constituye un verdadero problema de salud. Se estima que cerca de 4 millones de muertes al año están relacionadas directamente con esta afección, avalado por los cambios en los estilos de vida de la población, asociados al sedentarismo, la obesidad, la hipertensión arterial y otros factores de riesgo cardiovasculares.(Vintimilla Enderica, Mendoza, YO, Motoche Apolo, \& Ortega Flores, 2019). La prevalencia de la diabetes tipo 2 sigue aumentando entre los niños y adolescentes obesos, pero existen pocos tratamientos. La cirugía bariátrica ha emergido como un tratamiento potencial para la obesidad debido a que causa reducción de peso sustancial y duradero. Los datos recientes sugieren que la cirugía bariátrica puede ser eficaz en el tratamiento de la diabetes tipo 2. En este artículo se revisa la carga y las complicaciones de la diabetes tipo 2 en adolescentes, analizar los resultados y las complicaciones de la cirugía bariátrica entre los jóvenes con diabetes tipo 2, y concluir con recomendaciones para futuros criterios de investigación y el potencial para el uso de la cirugía bariátrica en adolescentes con diabetes tipo 2.

Antes de 1992, la diabetes tipo 2 representaba el 4\% de los nuevos diagnósticos de diabetes en adolescentes(Pinhas-Hamiel, y otros, 1996). Estimaciones recientes sugieren que ahora casi la mitad de los nuevos casos de diabetes en los adolescentes puede ser denominado diabetes tipo 2 (Dabelea, Mayer-Davis, \& Saydah, 2014). El aumento en el tipo de incidente 2 diabetes entre los jóvenes sigue en gran parte el aumento de la obesidad infantil, pero los datos recientes sugieren que otros factores, como el crecimiento de las poblaciones étnicos minoritarios en los EE.UU., la exposición a la diabetes materna en el útero, los disruptores endocrinos en el medio ambiente y 


\section{Bypass gástrico en el tratamiento de diabetes tipo 2 en la juventud}

Vol. 3, núm. 3., (2019)

Andrea Geraldine Castro Maquilón; Charles David Altamirano Olvera; Thalía Jocelyn Medina Checa; Juan Sebastián de Mora Flores

aumento de la conciencia y la detección más comunes de los niños y adolescentes, puede ser importante.

La Diabetes Juvenil en el estudio de una investigación observacional de la incidencia y prevalencia de la diabetes pediátrica, se proyecta que para el 2050 cerca de 85.000 adolescentes solo en los EE.UU. se verán afectados por la diabetes tipo 2 (Dabelea, Mayer-Davis, \& Saydah, 2014). Ha habido un aumento paralelo de la Auditoría Nacional del Reino Unido 2013-2014 en la Diabetes Pediátrica (NPDA) que incluye a pacientes de hasta 19 años que observó 500 casos de diabetes tipo 2, un marcado aumento en la incidencia desde el año 2000 cuando se describieron los primeros casos reportados en Birmingham, Inglaterra (Ehtisham, Barrett, \& Shaw, 2000). Del mismo modo, los datos de la India yJapón muestran creciente incidencia de diabetes tipo 2 entre los jóvenes. En 2006-2009, casi el 50\% de la diabetes juvenil de inicio (25 años) se atribuyó a la diabetes tipo 2 en la India y en Japón, la diabetes tipo 2 se diagnostica ahora dos veces más que la diabetes tipo 1 entre los adolescentes. Estos estudios auguran consecuencias importantes para la salud pública como los jóvenes con diabetes tipo 2 de desarrollar comorbilidades a un ritmo más rápido que las personas con diabetes tipo 1 y están en un riesgo acelerado para desarrollar enfermedad arterial coronario.

Mientras que la progresiónanormal del metabolismo de la glucosa para desarrollar la diabetes tipo 2 en adultos se produce normalmente durante años o décadas, la aparición de la diabetes tipo 2 en la adolescencia sugiere una patogénesis más agresiva. El fracaso del tratamiento de prueba se define como un HbAlc mayor de 0,8\% durante 6 meses o descompensación metabólica que requieren terapia con insulina (Zeitler, Epstein, \& Grey, 2007). Las tasas de fracaso del tratamiento fueron altas en los tres brazos del ensayo como 52, 39, y 47\% de los participantes 


\section{Bypass gástrico en el tratamiento de diabetes tipo 2 en la juventud}

Vol. 3, núm. 3., (2019)

Andrea Geraldine Castro Maquilón; Charles David Altamirano Olvera; Thalía Jocelyn Medina Checa; Juan Sebastián de Mora Flores

no metformina sola, metformina más rosiglitazona y metformina más el estilo de vida de intervención, respectivamente, con un tiempo medio de falla de 11 meses. Además, ninguno de los regímenes de tratamiento mejorado o función de las células B pancreáticas estabilizadas se estimaron a partir de las pruebas de tolerancia a la glucosa por vía oral; de hecho, la función global de las células B se redujo en un 20-35\% por año en la cohorte. En particular, para los que no pudieron mantener el control glucémico, la función de las células B era 40-50\% más bajos que al inicio del estudio en los que no progresan a que requiere tratamiento con insulina (TODAY Study Group., 2013). Este curso de la progresión de la diabetes parece ser diferente a lo que se ve típicamente en adultos con diabetes de tipo 2 de inicio.

\section{Metodología.}

Para el desarrollo de este proceso investigativo, se plantea como metodología la encaminada hacia una orientación científica particular que se encuentra determinada por la necesidad de indagar en forma precisa y coherente una situación, en tal sentido (Davila, 2015) define la metodología "como aquellos pasos previos que son seleccionados por el investigador para lograr resultados favorables que le ayuden a plantear nuevas ideas".(p.66)

Lo citado por el autor, lleva a entender que el desarrollo de la acción investigativa busca simplemente coordinar acciones enmarcadas en una revisión bibliográfica con el fin de complementar ideas previas relacionadas a la cirugía bariátrica en el tratamiento de la diabetes tipo 2 a través de una revisión de literatura, para así finalmente elaborar un cuerpo de consideraciones generales que ayuden a ampliar el interés propuesto. 


\section{Bypass gástrico en el tratamiento de diabetes tipo 2 en la juventud}

Vol. 3, núm. 3., (2019)

Andrea Geraldine Castro Maquilón; Charles David Altamirano Olvera; Thalía Jocelyn Medina Checa; Juan Sebastián de Mora Flores

\section{Tipo de Investigación}

Dentro de toda práctica investigativa, se precisan acciones de carácter metodológico mediante las cuales, se logra conocer y proyectar los eventos posibles que la determinan, así como las características que hacen del acto científico un proceso interactivo ajustado a una realidad posible de ser interpretada. En este sentido, se puede decir, que la presente investigación corresponde al tipo documental, definido por Castro (2016), "se ocupa del estudio de problemas planteados a nivel teórico, la información requerida para abordarlos se encuentra básicamente en materiales impresos, audiovisuales y /o electrónicos". (p.41).

En consideración a esta definición, la orientación metodológica permitió la oportunidad de cumplir con una serie de actividades inherentes a la revisión y lectura de diversos documentos donde se encontraron ideas explicitas relacionadas con los tópicos encargados de identificar a cada característica insertada en el estudio. Por lo tanto, se realizaron continuas interpretaciones con el claro propósito de revisar aquellas apreciaciones o investigaciones propuestas por diferentes investigadores relacionadas con el tema de interés, para luego dar la respectiva argumentación a los planteamientos, en función a las necesidades encontradas en la indagación.

\section{Fuentes Documentales}

El análisis correspondiente a las características que predomina en el tema seleccionado, llevan a incluir diferentes fuentes documentales encargadas de darle el respectivo apoyo y en ese sentido cumplir con la valoración de los hechos a fin de generar nuevos criterios que sirven de referencia a otros procesos investigativos. Para (CASTRO, 2016) las fuentes documentales incorporadas en la investigación documental o bibliográfica, "representa la suma de materiales 


\section{Bypass gástrico en el tratamiento de diabetes tipo 2 en la juventud}

Vol. 3, núm. 3., (2019)

Andrea Geraldine Castro Maquilón; Charles David Altamirano Olvera; Thalía Jocelyn Medina Checa; Juan Sebastián de Mora Flores

sistemáticos que son revisados en forma rigurosa y profunda para llegar a un análisis del fenómeno".(p.41). Por lo tanto, se procedió a cumplir con la realización de una lectura previa determinada para encontrar aquellos aspectos estrechamente vinculados con el tema, con el fin de explicar mediante un desarrollo las respectivas apreciaciones generales de importancia.

\section{Técnicas para la Recolección de la Información}

La conducción de la investigación para ser realizada en función a las particularidades que determinan a los estudios documentales, tiene como fin el desarrollo de un conjunto de acciones encargadas de llevar a la selección de técnicas estrechamente vinculadas con las características del estudio. En tal sentido, (Bolívar, 2015), refiere, que es "una técnica particular para aportar ayuda a los procedimientos de selección de las ideas primarias y secundarias”. (p. 71).

Por ello, se procedió a la utilización del subrayado, resúmenes, fichaje, como parte básica para la revisión y selección de los documentos que presentan el contenido teórico. Es decir, que mediante la aplicación de estas técnicas se pudo llegar a recoger informaciones en cuanto a la revisión bibliográfica de los diversos elementos encargados de orientar el proceso de investigación. Tal como lo expresa, (Bolívar, 2015) "las técnicas documentales proporcionan las herramientas esenciales y determinantes para responder a los objetivos formulados y llegar a resultados efectivos" (p. 58). Es decir, para responder con eficiencia a las necesidades investigativas, se introdujeron como técnica de recolección el método inductivo, que hizo posible llevar a cabo una valoración de los hechos de forma particular para llegar a la explicación desde una visión general.

Asimismo, se emplearon las técnicas de análisis de información para la realización de la investigación que fue ejecutada bajo la dinámica de aplicar diversos elementos encargados de 


\section{Bypass gástrico en el tratamiento de diabetes tipo 2 en la juventud}

Vol. 3, núm. 3., (2019)

Andrea Geraldine Castro Maquilón; Charles David Altamirano Olvera; Thalía Jocelyn Medina Checa; Juan Sebastián de Mora Flores

determinar el camino a recorrer por el estudio, según, (Bolívar, 2015) las técnicas de procesamiento de datos en los estudios documentales "son las encargadas de ofrecer al investigador la visión o pasos que debe cumplir durante su ejercicio, cada una de ellas debe estar en correspondencia con el nivel a emplear" (p. 123). Esto indica, que para llevar a cabo el procesamiento de los datos obtenidos una vez aplicado las técnicas seleccionadas, tales como: fichas de resumen, textual, registros descriptivos entre otros, los mismos se deben ajustar al nivel que ha sido seleccionado.

\section{Resultados.}

\section{Complicaciones de Diabetes Tipo 2}

Las complicaciones de la diabetes tipo 2 en adultos están bien documentadas e incluyen infarto de miocardio, derrame cerebral, falla renal, neuropatía, retinopatía y enfermedad vascular periferica. Casi el $70 \%$ de pacientes adultos con diabetes tipo 2 sucumben a la muerte por enfermedad cardiovascular. La diabetes tipo 2 en adultos también es responsable de más casos de fracaso renal y la enfermedad vascular periférica que conduce a la amputación que cualquier otra enfermedad. A pesar de las complicaciones a largo plazo de la diabetes tipo 2 de inicio adolescente aún no están claras, la carga de riesgo cardiovascular esta así documentado y progresa rápidamente con el tiempo.

Con el uso de marcadores no invasivos de aterosclerosis temprana que predicen futuro infarto de miocardio y accidente cerebrovascular, (Urbina, Kimball, Khoury, Daniels, \& Dolan, 2010) han encontrado que los jóvenes con diabetes tipo 2 (edad media 18 años) han aumentado el grosor medio de la carótida y rigidez periférica vascular en comparación con la edad de razas 


\section{Bypass gástrico en el tratamiento de diabetes tipo 2 en la juventud}

Vol. 3, núm. 3., (2019)

Andrea Geraldine Castro Maquilón; Charles David Altamirano Olvera; Thalía Jocelyn Medina Checa; Juan Sebastián de Mora Flores

delgadas y obesas. Mayor masa ventricular izquierda y peor función diastólica también se encontraron en estos jóvenes y se asociaron con mayores presiones sanguíneas, índice de masa corporal, una mayor duración de la diabetes, y peor control glucémico. Otros han demostrado que complicaciones renales también se acumulan mucho más temprano para los jóvenes con diabetes tipo 2, en comparación con la diabetes tipo 1. Aproximadamente el $6 \%$ de adolescentes con diabetes tipo 2 desarrollan insuficiencia renal por la edad de 20 años (dentro de los 5 años del diagnóstico de la diabetes), y por edad 29 años, 2.3\% han desarrollado la enfermedad renal en etapa terminal (Dart, y otros, 2012). También hay pruebas de que los jóvenes con diabetes tipo 2 han disminuido la función cognitiva, el volumen del cerebro, y los cambios microestructurales en la materia blanca en comparación con los sujetos a control de obesidad.

La piedra angular de la medicina en gestionar la unificación de los jóvenes obesos con diabetes tipo 2 es la intervención del estilo de vida como la dieta y el ejercicio. Sin embargo, la evidencia demuestra que este enfoque no es el éxito en la mayoría de los pacientes, y la mayoría de los pacientes requieren terapia de farmacológica adyuvante con metformina y / o insulina subcutánea. Desafortunadamente, la metformina es a menudo ineficaz para mejorar el control glucémico o para limitar el aumento de peso, y la insulina promueve el aumento de peso (Zeitler, Epstein, \& Grey, 2007). Como resultado, los procedimientos de pérdida de peso quirúrgicos están siendo examinados como alternativas potencialmente atractivas para el tratamiento de la obesidad y la diabetes tipo 2 en adolescentes. 


\section{Bypass gástrico en el tratamiento de diabetes tipo 2 en la juventud}

Vol. 3, núm. 3., (2019)

Andrea Geraldine Castro Maquilón; Charles David Altamirano Olvera; Thalía Jocelyn Medina Checa; Juan Sebastián de Mora Flores

\section{Tratamiento Quirúrgico de la Obesidad y Diabetes Tipo 2}

Hay una base emergente de información científica que proporciona evidencia de la efectividad de la cirugía bariátrica para tratar la diabetes, así como la obesidad en pacientes adultos. Los sujetos obesos suecas (SOS) es un estudio de intervención controlado que ha proporcionado algunos de los datos más importantes sobre los resultados de salud y mortalidad a largo plazo para adultos con obesidad severa tratados con cirugía bariátrica o la atención médica de rutina (sujetos de control). El grupo de estudio ha informado de resultados durante un período de 14 a 27 años y ha demostrado, por ejemplo, una duradera reducción del $25 \%$ en el peso corporal y la reducción de $30 \%$ en mortalidad en más de 20 años para los participantes que tenían Bypass gástrico. Para los participantes con diabetes tipo 2 al inicio del estudio, la tasa de remisión a los 15 años para los que no se someten a cirugía fue de 6,5\%. Para aquellos que se sometieron a cirugía, la remisión se observó en el 30\%. Es importante destacar que el riesgo de desarrollar complicaciones microvasculares de la diabetes en el grupo quirúrgico fue de aproximadamente la mitad de la observada en el grupo de control que no tiene la cirugía (Sjostrom, Peltonen, \& Jacobson, 2014). Varios ensayos más de corto plazo controlados aleatoriamente de la cirugía bariátrica para el tratamiento de la diabetes tipo 2 en adultos también han demostrado tasas alentadoras de remisión iniciales de casi el 50\% a los 3 años después de la derivación gástrica. Aunque también ha habido una recurrencia lento y constante de la diabetes en algunos pacientes quirúrgicos que inicialmente experimentan remisión, las tasas de diabetes no se acercan a los niveles en los sujetos controlados no operados (Arterburn, Bogart, \& Sherwood, 2013).

Durante la última década, los datos demostraron mejoras espectaculares en peso y el riesgo cardiometabólico para los jóvenes sometidos a una variedad de operaciones bariátricas para 


\section{Bypass gástrico en el tratamiento de diabetes tipo 2 en la juventud}

Vol. 3, núm. 3., (2019)

Andrea Geraldine Castro Maquilón; Charles David Altamirano Olvera; Thalía Jocelyn Medina Checa; Juan Sebastián de Mora Flores

perdida de peso y otros métodos también han surgido. Los resultados de los primeros grandes Institutos Nacionales de Salud multicéntrico patrocinado, estudio prospectivo de la cirugía bariátrica en adolescentes y adultos jóvenes fueron publicados recientemente (Inge, Courcoulas, \& Jenkins, 2016). Los participantes en la evaluación del estudio longitudinal adolescente de la cirugía bariátrica (Teen-LABS) fueron una edad media de 17 años al inicio del estudio antes de la cirugía. Esta cohorte experimentó una reducción del $31 \%$ en peso a 1 año (similartanto para la derivación gástrica y la mangagástrica) que fue sólo ligeramente readquirida por año 2 y 3. En elaño 3 , la pérdida media por ciento en peso fue de $28 \%$ en el grupo que fue sometido a by-pass gástrico y $26 \%$ en el grupo sometido a la manga gástrica. Durabilidad de la pérdida de peso también se ha demostrado en los niños, de 14 años de edad después de la manga gástrica. En años postoperatorio, los pacientes tenían una pérdida media de IMC de $15 \mathrm{~kg} / \mathrm{m} 2$, (Alqahtani, Elahmedi, \& Qahtani, 2016).

El estudio adolescent-LABS encontró una remisión 74\% en la hipertensión, $66 \%$ de remisión de la dislipidemia, y 86\% de resolución de la función renal anormal (definido por la tasa de filtración glomerular baja o proteínaUría) (Inge, Courcoulas, \& Jenkins, 2016). Del mismo modo, incluye apnea obstructiva del sueño, síndrome de ovario poliquístico, y el hígado graso, también se han informado de mejoras en otras comorbilidades relacionadas con la obesidad.

También se observaron mejoras en la glucosa y el metabolismo después de una cirugía de bypass gástrico en adolescentes con y sin diabetes tipo 2. Uso de pruebas a tolerancia a la glucosa intravenosa, las medidas de la secreción de insulina (la respuesta aguda de insulina a intravenosa 


\section{Bypass gástrico en el tratamiento de diabetes tipo 2 en la juventud}

Vol. 3, núm. 3., (2019)

Andrea Geraldine Castro Maquilón; Charles David Altamirano Olvera; Thalía Jocelyn Medina Checa; Juan Sebastián de Mora Flores

glucosa), sensibilidad a la insulina, y el índice dedisposición (una estimación de la función de células b corregido para sensibilidad a la insulina predominante) se obtuvieron antes y después de la derivación gástrica laparoscópica en 22 jóvenes sin diabetes tipo 2 y 2 jóvenes con debates tipo

2. Al inicio del estudio, todos los jóvenes presentaron marcada sensibilidad a la insulina (aproximadamente un tercio de la de los adolescentes delgados) y los valores de índice con deficiencias disposición función de las células b que eran aproximadamente la mitad que informan de opinión en adolescentes delgados. En el año después de un bypass gástrico, los adolescentes que no tienen diabetes demostraron un aumento de la sensibilidad a la insulina de tres a cuatro veces, mientras que el índice de disposición mejorada por doble. La secreción proinsulina, un measeguro de la disfunción de las células $\mathrm{B}$, también se redujo significativamente. En tono rimbombante, para los dos adolescentes con diabetes tipo 2 tratados con bypass gástrico, hubo una clara evidencia que tanto la sensibilidad a la insulina y la función de las células B, pero mejoraron en mayor medida en el adolescente que aún no habían requerido insulina exógena al inicio del estudio. Tomados en conjunto, estos datos fisiológicos demuestran que el bypass gástrico en adolescentes no sólo invierte la profunda resistencia a la insulina asociada con obesidad severa, pero también puede mejorar notablemente la célula B. La "fatiga" que es característico de personas que también son considerados como de alto riesgo de franca progresión a la diabetes tipo 2 (Beamish, D’Alessio, \& Inge, 2015).

Varios otros estudios han informado de los resultados clínicos y metabólicos de adolescentes con diabetes tipo 2 después de la cirugía bariátrica. Sin embargo, cada uno ha incluido muy pocos participantes con diabetes tipo 2, y las definiciones de los casos utilizadas para la 


\section{Bypass gástrico en el tratamiento de diabetes tipo 2 en la juventud}

Vol. 3, núm. 3., (2019)

Andrea Geraldine Castro Maquilón; Charles David Altamirano Olvera; Thalía Jocelyn Medina Checa; Juan Sebastián de Mora Flores

diabetes tipo 2 al inicio del estudio y la metodología utilizada para evaluar la respuesta a la cirugía no se estandarizaron, por lo que se hace difícil realizar inferencias.

Las complicaciones potenciales de cirugía para perder peso en la juventud.

Aunque las tasas de remisión de la diabetes tipo 2 en los jóvenes después de la cirugía bariátrica parecen altas, existen claros riesgos de la cirugía, incluyendo cirugía ycomplicaciones nutricionales que deben ser considerados cuidadosamente. En 2013, se informaron los resultados quirúrgicos de un solo hospital de niños que incluyó 77 procedimientos de derivación gástrica consecutivos en adolescentes obesos (Miyano, Jenkins, Xanthakos, Garcia, \& Inge, 2013). Se observaron complicaciones intraoperatorias en $3 \%$ de los jóvenes, no se observaron complicaciones perioperatorias (definidos como una complicación dentro de los 30 días) en 22\% y $13 \%$ tienen una complicación entre los 31 y 90 días. Las complicaciones postoperatorias más comunes incluyen estenosis gastroyeyunal anastomótica (17\%), reoperación (13\%), fugas (7\%), y la deshidratación (7\%).

Las deficiencias nutricionales, se ven comúnmente como vitamina B12, La deficiencia de tiamina y vitamina $\mathrm{D}$, son también un importante potencial a corto y largo plazo en complicaciones de la cirugía bariátrica en adolescentes(Inge, Courcoulas, \& Jenkins, 2016). La recaída de diabetes tipo 2 es otra posible complicación después de la cirugía bariátrica . Los datos retrospectivos en adultos sugieren que hasta un tercio de los adultos experimentan una recaída dentro de los 5 años de remisión inicial. La recaída en adultos estáasociadocon la recuperación del peso, mayor duración de la diabetes, y el uso de insulina antes de la cirugía (Brethauer, Aminian, \& RomeroTalamas, 2013) . 


\section{Bypass gástrico en el tratamiento de diabetes tipo 2 en la juventud}

Vol. 3, núm. 3., (2019)

Andrea Geraldine Castro Maquilón; Charles David Altamirano Olvera; Thalía Jocelyn Medina Checa; Juan Sebastián de Mora Flores

Por lo tanto, al considerar los procedimientos de pérdida de peso quirúrgicos como un tratamiento potencial de la diabetes tipo 2, los riesgos de la cirugía deben ser considerados a fondo. Charlas que informen a los pacientes y sus familias acerca de las posibles complicaciones quirúrgicas y nutricionales, así como potencial recaída de diabetes son enormemente importantes.

\section{Momento óptimo de la intervención quirúrgica para diabetes tipo 2 en la juventud.}

Para los adultos, la Federación Internacional de diabetesen declaración 2011 recomienda la cirugía para bajar de peso debe ser una prioridadpara los pacientes con diabetes tipo 2 que tienen un índice de masa corporal superior a $40 \mathrm{~kg} / \mathrm{m} 2$. La cirugía también está indicado para pacientes con un IMC de 35 a $40 \mathrm{~kg} / \mathrm{m} 2$ y la diabetes mal controlada (HbA1c.7.5\%) a pesar de la terapia convencional totalmente optimizado. La declaración también informa consideración de la cirugía para los pacientes con un IMC de 30-35 kg / m2 si el objetivo de $\mathrm{HbA} 1 \mathrm{c}(, 7,5 \%)$ no se alcanza y si otras comorbilidades referentes a la obesidad están presentes. En los adolescentes, las indicaciones para el uso de la cirugía para bajar de peso en pacientes con diabetes tipo 2 han sido algo menos estrictos. La cirugía ha sido reconsiderada a aquellos que cumplen criterios clínicos para la diabetes tipo 2 y la obesidad grave (definida como un absoluto IMC $>35 \mathrm{~kg} / \mathrm{m} 2 \mathrm{o}$ un IMC $>120 \%$ del percentil 95 para la edad y sexo) (Pratt, Lenders, \& Dionne, 2009). Aunque este es un punto de partida razonable, recomendaciones de tratamiento individualizados (o momento del tratamiento), teniendo en cuenta factores biológicos pueden ser preferible desde el punto de vista de riesgo-beneficio en el futuro. 


\section{Bypass gástrico en el tratamiento de diabetes tipo 2 en la juventud}

Vol. 3, núm. 3., (2019)

Andrea Geraldine Castro Maquilón; Charles David Altamirano Olvera; Thalía Jocelyn Medina Checa; Juan Sebastián de Mora Flores

\section{Conclusiones.}

La evidencia acumulada sugiere que la diabetes tipo 2 en adolescentes progresa rápidamente y es más agresivo que la diabetes tipo 2 en adultos. La diabetes tipo 2 en los resultados de la juventud en múltiples sistemas crea daño de órgano con el tiempo, probablemente el resultado combinado de hiperglicemia, hipertensión y dislipidemia entre otros factores de riesgo. Los datos de estudios clínicos demuestran sustancialmejora en la resistencia a la insulina y función de las células b en la juventud severamente obesa sin diabetes después de un bypass gástrico, y varios estudios de observación también demuestran altas tasas de remisión de la diabetes tipo 2 en la juventud. Estas observaciones apoyan las recomendaciones para la gestión de cirugía bariátrica de los adolescentes con obesidad severa adecuadamente seleccionados con diabetes tipo 2. Las decisiones con respecto momento óptimo de la cirugía para el máximo impacto sobre la diabetes tipo 2 deben tratar de preservar la función secretora de las células b.

\section{Bibliografía.}

Alqahtani, A., Elahmedi, M., \& Qahtani, A. (2016). Laparoscopic sleeve gastrectomy in children younger than 14 years: refuting the concerns. Ann Surg , 312-319.

Arterburn, D., Bogart, A., \& Sherwood, N. (2013). A multisite study of long-term remission and relapse of type 2 diabetes mellitus following gastric bypass. Obes Surg , 93-102.

Beamish, A., D’Alessio, D., \& Inge, T. (2015). Controversial issues: when the drugs don't work, can surgery provide a different outcome for diabetic adolescents? Surg Obes Relat Dis, 946-950.

Bolívar, J. (2015). Investigación Documental. México. Pax.

Brethauer, S., Aminian, A., \& Romero-Talamas, H. (2013). Can diabetes be surgically cured? Long-term metabolic effects of bariatric surgery in obese patients with type 2 diabetes mellitus. Ann Surg, 628-636. 


\section{Bypass gástrico en el tratamiento de diabetes tipo 2 en la juventud}

Vol. 3, núm. 3., (2019)

Andrea Geraldine Castro Maquilón; Charles David Altamirano Olvera; Thalía Jocelyn Medina Checa; Juan Sebastián de Mora Flores

Castro, J. (2016). Técnicas Documentales. México. Limusa.

Dabelea, D., Mayer-Davis, E., \& Saydah, S. (2014). SEARCH for Diabetes in Youth Study. Prevalence of type 1 and type 2 diabetes among children and adolescents from 2001 to 2009. JAMA , 1778-1786.

Dart, A., Sellers, E., Martens, P., Rigatto, C., Brownell, M., \& Dean, H. (2012). High burden of kidney disease in youth-onset type 2 diabetes. . Diabetes Care, 1265-1271.

Davila, A. (2015). Concepto de terminos cientificos. Caracas: Oasis.

Ehtisham, S., Barrett, T., \& Shaw, N. (2000). Type 2 diabetes mellitus in UK children: an emerging problem. Diabet Med, 867-871.

Inge, T., Courcoulas, A., \& Jenkins, T. (2016). Weight loss and health status 3 years after bariatric surgery in adolescents. $N$ Engl J Med, 113-123.

Miyano, G., Jenkins, T., Xanthakos, S., Garcia, V., \& Inge, T. (2013). Perioperative outcome of laparoscopic Roux-en-Y gastric bypass: a children's hospital experience. J Pediatr Surg , 2092-2098.

Pinhas-Hamiel, O., Dolan, L., Daniels, S., Standiford, D., Khoury, P., \& Zeitler, P. (1996). Increased incidence of non-insulin-dependent diabetes mellitus among adolescents. $J$ Pediatr, 608-615.

Pratt, J., Lenders, C., \& Dionne, E. (2009). Best practice updates for pediatric/adolescent weight loss surgery. Obesity (Silver Spring), 901-910.

Sjostrom, L., Peltonen, M., \& Jacobson, P. (2014). Association of bariatric surgery with long-term remission of type 2 diabetes and with microvascular and macrovascular complications. JAMA, 2297-2304.

TODAY Study Group. (2013). Effects of metformin, metformin plus rosiglitazone, and metformin plus lifestyle on insulin sensitivity and b-cell function in TODAY. Diabetes Care , 17491757.

Urbina, E., Kimball, T., Khoury, P., Daniels, S., \& Dolan, L. (2010). Increased arterial stiffness is found in adolescents with obesity or obesity related type 2 diabetes mellitus. J Hypertens, 1692-1698.

Vintimilla Enderica, P., Mendoza, G., YO, Motoche Apolo, K., \& Ortega Flores, J. (2019). Diabetes Mellitus Tipo 2: Incidencias, Complicaciones y Tratamientos Actuales. RECIMUNDO, https://doi.org/10.26820/recimundo/3.(1).enero.20.

Zeitler, P., Epstein, L., \& Grey, M. (2007). Treatment options for type 2 diabetes in adolescents and youth: a study of the comparative efficacy of metformin alone or in combination with

Revista Científica Mundo de la Investigación y el Conocimiento. 3 (3). pp. 145-162 


\section{Bypass gástrico en el tratamiento de diabetes tipo 2 en la juventud}

Vol. 3, núm. 3., (2019)

Andrea Geraldine Castro Maquilón; Charles David Altamirano Olvera; Thalía Jocelyn Medina Checa; Juan Sebastián de Mora Flores

rosiglitazone or lifestyle intervention in adolesc with type 2 diabetes. . Pediatr Diabetes, 74-87. 